Jorge Villegas 1

Mariano Noriega 1

Susana Martínez 1

Sandra Martínez 1

\title{
Trabajo y salud en la industria maquiladora mexicana: una tendencia dominante en el neoliberalismo dominado
}

\author{
Work and health in the Mexican "maquiladora" \\ industry: a dominant trend in dominated \\ neoliberalism
}

1 Universidad Autónoma Metropolitana, Xochimilco. Calz. del Hueso 1100, Del. Coyoacán,

México, D.F., México. jvillega@cueyatl.uam.mx

\begin{abstract}
This paper discusses health and working conditions of workers in the export-oriented "maquiladora" industry in Mexico. The work is intensive and performed mostly by women. It is the country's second largest source of foreign exchange. The "maquiladora" industry illustrates the effect of neoliberalism on working and health conditions, unveiling the impact of overall flexibilization of labor and growing technological changes. The situation for risks and demands is complex. Taylorist demands persist in work organization, with multiple chemical risks and probable effects beyond the workplace. There are also demands from repetition strain and uncomfortable positions. Effects on workers' health include high morbidity rates as reflected by demands on the social health/welfare services, some two to four times greater than for other industrial workers. There is a high percentage of stress-related illnesses as the reason for medical consultation. There are also effects on workers' families. Low birthweight is 2.8 times higher in women who work in the "maquila" than in other labor groups. Work-related accidents are common, the second most frequent cause for medical consultation in some cases. The analysis identifies some of the tendencies in which workers' health could be important in coming years for large contingents of workers. Key words Worker's Health; Working Conditions; Occupational Health
\end{abstract}

Resumen Se analizan las condiciones laborales y de salud de los trabajadores de la Industria Maquiladora de Exportación en México. Hace uso intensivo de la fuerza de trabajo mayoritariamente femenina,constituye la segunda fuente de divisas. Ilustra una tendencia dominante en el neoliberalimo respecto a condiciones de trabajo y salud, permite apreciar el impacto de la generalizada flexibilización laboral y del creciente cambio tecnológico. El panorama de los riesgos y exigencias es complejo. En la organización laboral persisten exigencias del taylorismo, los riesgos químicos son múltiples y con probables efectos que rebasan el ámbito laboral, también existen exigencias de trabajo repetitivo y posiciones incómodas. Las repercusiones en la salud muestran elevados índices de morbilidad por demanda en los servicios de seguridad social, entre 2 y 4 veces más altos que para otros trabajadores industriales. Padecimientos asociados al estrés ocupan elevado porcentaje entre los motivos de consulta. También se muestran repercusiones en las familias, el bajo peso al nacer de los hijos es un riesgo 2.8 veces mayor para obreras de la maquila respecto de otros grupos de trabajadoras. Los accidentes laborales alcanzan cifras elevada y llegando a ser la segunda causa de consulta en algunos casos. Este análisis permite identificar algunas tendencias que en la salud laboral pueden ser importantes en los próximos años para amplios grupos de trabajadores. Palabras clave Salud de los Trabajadores; Condiciónes del Trabajo; Salud Ocupacional 


\section{Introducción}

Las diferentes manifestaciones del neoliberalismo en México - globalización, flexibilidad, “terciarización” de la economía, precarización del empleo, entre otras - han originado modificaciones profundas en las condiciones de vida, de trabajo y de salud de la mayoría de los trabajadores mexicanos.

El nuevo modelo "modernizador" no sólo se reduce a los aspectos de productividad, sino que determina - o por lo menos influye de manera decisiva - los terrenos político, sindical, ideológico, cultural, científico y trastoca de manera central las condiciones de reproducción de los trabajadores. El propósito de este documento es analizar las características que asume este proceso en una de las áreas más dinámicas de la economía mexicana, la industria maquiladora de exportación IME, y el impacto que este fenómeno tiene en las condiciones laborales y de salud de los trabajadores de ese sector. Este análisis permite identificar además, algunas tendencias que en el terreno de la salud laboral parecen ser importantes en los próximos años para amplios grupos de trabajadores, en la medida en que el modelo modernizador tiende a generalizarse a los diversos sectores de la economía. El estudio de esta situación es además importante porque la IME ha llamado la atención por los efectos negativos que ha producido en las condiciones de vida, de trabajo y de salud de cada vez mayor número de obreros.

\section{Origen y desarrollo de la maquila}

Desde la segunda mitad del siglo XX se ha efectuado una revolución tecnológica de primera importancia que, entre otras cosas, ha satisfecho una vieja aspiración capitalista, la transferencia de fases de producción de las economías centrales a países de menor desarrollo. Las Zonas de Producción para la Exportación, en las cuales millones de obreros ensamblan productos para el mercado mundial, son las manifestaciones más claras de estas tendencias y se ubican en Asia, América Latina, el Caribe, y cada vez en mayor grado, en otras partes del mundo (Fernández, 1986).

En México este fenómeno lo constituye la IME, la cual está conformada por el conjunto de empresas o establecimientos que se dedican a realizar alguna o varias de las etapas del proceso productivo, generalmente de ensamblado o de tipo administrativo. La actividad de maquila está destinada a la transformación, ela- boración y reparación de mercancías de procedencia extranjera, importadas temporalmente, para su posterior exportación. También contempla aquellas actividades de servicio que tengan como finalidad la exportación o el apoyo a ésta (INEGI, 1994).

Para cumplir con estas características, una maquiladora importa temporalmente partes, piezas o componentes de una empresa extranjera, la cual funge como cliente de la propia maquiladora, y efectúa la devolución de dichos materiales, una vez que han sido incorporados a la manufactura de exportación. Una de las principales características de esta actividad es la utilización intensiva de mano de obra, lo cual genera una significativa cantidad de empleos en los lugares donde se instalan. Hace poco más de 30 años que se establecieron oficialmente las primeras empresas maquiladoras en México. Desde entonces, y a través de diferentes políticas de apertura económica instrumentadas por el Estado, el crecimiento de la industria maquiladora llegó a ser en 1993, después del petróleo, la segunda fuente generadora de divisas para el país (Espinosa-Torres et al., 1994). Actualmente más de $80 \%$ de las maquiladoras son de capital norteamericano y alrededor del $68 \%$ de la inversión en la zona de maquiladoras es norteamericana.

En su desarrollo se pueden distinguir diferentes etapas que le han dado su actual conformación (Kopinak, K. 1994). Durante los años 1965-1974, se instala y consolida la IME. Las leyes de México y Estados Unidos permitieron el nacimiento de las maquiladoras durante esa época, a lo largo de toda la frontera entre ambos países. Maquinarias, vehículos y componentes, fueron importadas a México, libres de impuestos, para ser ensamblados y luego enviados como producto final a Estados Unidos. México, sólo cobraba impuestos al valor agregado generado casi en su totalidad por la mano de obra. El programa estaba limitado a la región fronteriza norte donde el gobierno mexicano ya había experimentado con zonas de libre cambio en los años treinta.

Con las maquilas, México pretendía reemplazar los empleos perdidos debido a la terminación del programa Bracero, el cual desde 1942 había permitido que trabajadores mexicanos emigraran a Estados Unidos para trabajar temporalmente en la agricultura. La cancelación de Bracero coincidió con los esfuerzos de reestructuración de la empresa privada, al tratar de enfrentar la caída de sus ganancias. Este último factor ha sido el principal responsable del rápido crecimiento de la IME en México. 
Desde el principio parecía que la estrategia de usar maquiladoras para resolver los problemas de desempleo e inmigración había fallado, ya que las nuevas industrias no emplearon braceros como se esperaba, sino que prefirieron mujeres jóvenes sin experiencia laboral. Además, la existencia de las maquiladoras en el norte fue como un imán que atrajo inmigrantes del interior, que al no ser absorbidos por las maquiladoras incrementaron el desempleo local y la emigración hacia Estados Unidos (Kopinak, 1994). Cabe señalar desde ahora que la presencia predominante de la mujer en la fuerza de trabajo de la maquila, vendrá a ser uno de los rasgos distintivos de ésta y un elemento esencial a considerar en el análisis de los procesos de flexibilización laboral y en la problemática de las condiciones laborales y de salud.

El constante crecimiento de la maquila se detuvo en 1974 debido a la crisis que se produjo por la recesión en Estados Unidos. Durante los dos siguientes años, ocurre una pérdida importante de más de un tercio de los empleos. Ante esto, el gobierno mexicano se apresuró a eximir a las maquilas de algunos requisitos de la Ley Federal del Trabajo (LFT). El período de prueba para los trabajadores se extendió de 30 a 90 días. Las compañías también podían ajustar el tamaño de su fuerza laboral o aumentar las horas de trabajo de acuerdo a sus propias necesidades. Además se permitió el incremento de cláusulas unilaterales (propatronales) en los convenios colectivos que establecieron en forma explícita condiciones inferiores a las establecidas en la LFT. Es evidente que este tipo de apoyo a los empresarios significará para los trabajadores, la pérdida de importantes conquistas laborales y, en el terreno de la salud, la conformación de un escenario de condiciones laborales, riesgos y exigencias potencialmente nocivas (Denman, 1990).

Posteriormente, de 1977 a 1982, la maquila experimenta un crecimiento superior al de los primeros años, al tiempo que se inicia el abandono de la estrategia económica de substitución de importaciones, buscando un desarrollo a través de la exportación.

Con la crisis de la deuda externa en 1982, el Fondo Monetario Internacional presionó para que el país liberalizara su economía. Si bien la creación de empleos seguía siendo un objetivo importante, el gobierno favorecía ahora a las maquiladoras que contaban con mayor capital intensivo y menos mano de obra. Es al inicio de los ochenta cuando empieza un proceso de modernización en el que se da la “...incorporación de nuevas tecnologías, nuevas formas de organización del trabajo, cambios en la gestión de los recursos humanos y transformaciones en las relaciones contractuales" (Carrillo et al., 1992).

Como el peso seguía depreciándose, la mano de obra llegó a ser más barata que la de Corea del Sur, Taiwán, Hong Kong, Singapur y Brasil. Así, mientras se incrementaba el número de empleos en las maquiladoras, éstos tendían a costarles menos dólares a los inversionistas extranjeros y resultaban en salarios reales más bajos para los trabajadores mexicanos. Como resultado de esta política la fuerza laboral de las maquiladoras se triplicó. De hecho, los principales indicadores económicos de esta industria registraron en la década de los ochenta un acelerado ritmo de crecimiento, que supera en mucho el logrado durante los primeros años de su presencia en el país.

En 1990, cuando el gobierno mexicano solicitó a Estados Unidos ser incluido en el Tratado de Libre Comercio (TLC) consideró a la industria maquiladora como un elemento esencial de su política económica y fue designada como un instrumento necesario de ayuda para aumentar la competitividad internacional de la industria mexicana. Se veía la inversión extranjera como el principal medio para insertar a la economía nacional dentro de la economía global mundial y la conexión entre las dos esferas se haría a través de las maquiladoras.

En la actualidad, casi la mitad de las maquiladoras de la frontera norte se dedica al ensamblaje de equipos, aparatos, materiales electrónicos y eléctricos. Pero otras actividades importantes son el ensamblaje de equipo y materiales de transporte; la elaboración de productos derivados del petróleo, plásticos y químicos; la manufactura de productos metálicos y la producción de aparatos médicos, alimentos, ropa y textiles; si bien también existen algunas maquilas ubicadas en el sector servicios (Espinosa-Torres et al., 1994). En varias ciudades de la frontera constituyen el eje de la economía, involucrando elevados porcentajes de la mano de obra existente, entre 30 y $50 \%$ de la fuerza de trabajo de algunas ciudades (Balcazar et al., 1995).

En todo el país, aproximadamente 500,000 trabajadores laboran en alrededor de 2,000 establecimientos de esa industria; dos terceras partes de la fuerza laboral son mujeres (La Botz, 1994). Ellas se ubican principalmente en la maquila eléctrico-electrónica y del vestido, mientras que los hombres predominan en la producción de artículos de madera y metal (Espinosa-Torres et al., 1994).

Para establecer un balance acerca de las ventajas que representa "la vía maquiladora" 
para el desarrollo del país hay que tener presente las metas explícitas de los programas gubernamentales de apoyo a la IME: creación de empleos, elevación del nivel de calificación de los obreros, y transferencia de tecnología potencialmente importante para la industria nacional (Quintero, 1990). La industrialización fronteriza, lejos de proporcionar oportunidades ocupacionales a aquéllos que habían sido más afectados por la desaparición del Programa de Braceros (los trabajadores agrícolas), precipitó la incorporación de mujeres (especialmente jóvenes y solteras) a la fuerza de trabajo. Esto significó un mayor desempleo en los hombres y por lo tanto una mayor emigración hacia Estados Unidos. Asimismo el número de empleos generados no satisfizo la creciente demanda de trabajo, más aún cuando, en los últimos años, la IME presentó un ritmo de crecimiento descendente. En la Tabla 1, se observan tasas de crecimiento positivas en el período de 1985 a 1994, pero a partir de 1989 se nota un acelerado descenso. Si se toman los años de 1985 a 1988 se obtiene una tasa media de crecimiento del $21.1 \%$, mientras que para el lapso de 1989 a 1994, dicha tasa fue de sólo $5.15 \%$, a pesar del fuerte impulso otorgado por el gobierno dada su importancia en la generación de divisas.

Sobre la segunda y tercera metas, otra característica importante del Programa de Maquiladoras en México, aún cuando esta situación está cambiando como ya se mencionó, ha sido su extrema dependencia sobre las operaciones de trabajo intensivo, las cuales oficialmente están catalogadas como trabajo no calificado. Esto contradice de manera importante la suposición del gobierno de que esta forma de industrialización es un instrumento eficaz para elevar la destreza de los trabajadores y para transferir tecnología a la industria nacional.

Por otra parte, la IME continúa concentrándose en los estados fronterizos del norte, a pesar de que en 1989 se intentó fomentar la instalación de maquilas en cualquier parte del país. Así, tenemos que para 1980 se concentraba en tales entidades casi el $90 \%$ del personal ocupado por la IME, reduciéndose ligeramente para 1994 con el $87 \%$. Otra limitante ha sido el bajo porcentaje de bienes intermedios usados en la IME, que no sobrepasa el $2 \%$ del total de insumos de la maquila.

\section{Condiciones laborales y organización de los trabajadores en la maquila}

El fenómeno de las maquiladoras conlleva a una dinámica laboral nueva. No sólo significa la descentralización del proceso productivo en diferentes países periféricos, sino que implica un cambio en la estructura social y política del sistema capitalista. Por primera vez los elementos de producción, capital y trabajo, no están insertos en el mismo contexto espacial. De la misma manera, las negociaciones por mejores condiciones laborales no pueden ser el acuerdo tradicional entre el trabajador y/o el sindicato como representante laboral, y el patrón. Se conforma de esta manera, una nueva estructura ocupacional: por una parte, los patrones establecidos en las plantas matrices de los Estados Unidos o Japón y, por otra, un grupo trabajador que comprende obreros maqui-

Tabla 1

Empleos remunerados en la industria manufacturera 1985-1994.

\begin{tabular}{|c|c|c|c|c|c|c|}
\hline & \multicolumn{3}{|c|}{ indices promedio* } & \multicolumn{3}{|c|}{ tasas de crecimiento } \\
\hline & maquiladoras & n maquiladoras & total & maquiladoras & n maquiladoras & total \\
\hline 1985 & 100.0 & 100.0 & 100.0 & - & - & - \\
\hline 1986 & 117.5 & 99.0 & 100.3 & 17.50 & -1.00 & 0.35 \\
\hline 1987 & 145.5 & 100.0 & 103.3 & 23.83 & 1.01 & 2.96 \\
\hline 1988 & 177.6 & 99.8 & 105.5 & 22.06 & -0.20 & 2.09 \\
\hline 1989 & 201.2 & 101.2 & 108.5 & 13.29 & 1.40 & 2.86 \\
\hline 1990 & 215.5 & 101.3 & 109.6 & 7.11 & 0.10 & 1.05 \\
\hline 1991 & 223.2 & 99.9 & 108.9 & 3.57 & -1.38 & -0.67 \\
\hline 1992 & 240.1 & 96.2 & 106.7 & 7.56 & -3.67 & -1.99 \\
\hline 1993 & 257.4 & 90.0 & 102.2 & 7.19 & -6.49 & -4.25 \\
\hline 1994 & 258.7 & 89.5 & 101.8 & 0.52 & -0.56 & -0.37 \\
\hline
\end{tabular}

* $1985=100$

Fuente: Banco de México. Indicadores Económicos, México, 1995. 
ladores de los distintos países en los que se han instalado estas industrias. Mientras que los empresarios maquiladores cuentan con un dominio total de sus diferentes plantas en el mundo, los trabajadores se caracterizan por una desvinculación completa (Quintero, 1990).

En ese contexto, las posibilidades de defensa colectiva por parte de los trabajadores se ven seriamente disminuídas, el papel de los sindicatos se trastoca y pasan a ser, en muchos casos, defensores y promotores de las nuevas relaciones laborales. Tal es el caso del nuevo sindicalismo que ha sido considerado funcional y regresivo, porque atiende los intereses de las empresas e impide la organización democrática de los trabajadores, dejando éstos al margen de toda negociación (Carrillo et al., 1992). Al analizar los contratos colectivos se encuentra que, más que expresar las conquistas de los trabajadores y sus posibilidades de defensa, sancionan condiciones laborales en función de las nuevas necesidades de la producción, frecuentemente por abajo de los derechos reconocidos por la LFT, como ha sido demostrado al analizar diversos contratos colectivos vigentes en empresas maquiladoras en Chihuahua, una de las ciudades mexicanas donde se asienta un importante número de estas empresas (Sánchez, 1994).

Así, no sorprende que los descansos, permisos, vacaciones, estímulos y premios estén en función de las necesidades productivas. En lo que se refiere a la ejecución del trabajo, se regulan con mucha precisión todos los aspectos relacionados con la flexibilidad como: horarios, rotación de puestos, jornada y modificaciones en el sistema escalafonario. También se ha identificado que los contratos asumen la inestabilidad laboral y las formas de pago atadas a la productividad. Por el contrario, en cuanto a higiene y seguridad, las cláusulas son casi nulas, sólo se mencionan prácticas de primeros auxilios y servicios de enfermería. En esas circunstancias tampoco resulta extraño que en el Reglamento Interior de Trabajo de algunas empresas se incluyan sanciones y limitaciones de todo tipo, hasta moralistas y sexistas (Sánchez, 1994).

Los cambios tecnológicos y organizativos en los procesos laborales que se han señalado, conforman en la actualidad un complejo mosaico de situaciones que pueden ser entendidas en ciertos sentidos como una transición, pero también revelan las diversas alternativas por las que han optado las empresas y las posibilidades de defensa colectiva de los trabajadores. En este sentido se puede señalar que las estrategias de flexibilización se montan sobre es- tructuras y modelos aún vigentes y dominantes. Así, la flexibilidad laboral se está imponiendo como tendencia en los procesos productivos, pero conserva y generaliza estructuras organizativas anteriores como la taylorista, y aprovecha las nuevas propuestas de procesos que incorporan la automatización, la informática, la microelectrónica u otras propuestas ligadas a éstas como el control de calidad y la calidad total.

Si bien estas características pueden ser consideradas como una tendencia generalizada en el país, en la industria maquiladora se plantean con toda claridad. Así, en un estudio se reporta que, aunque se encuentran distintos tipos de regulación laboral, en el $47.9 \%$ de las plantas con avanzado desarrollo tecnológico predominan las prácticas laborales con normatividad integral, es decir, que tienen un reglamento interior de trabajo y un contrato colectivo, lo que sucede sólo en el $21.7 \%$ de los establecimientos con escaso desarrollo tecnológico. Es decir, aún cuando hay cambios tecnológicos importantes, los contratos colectivos pueden servir como instrumentos para flexibilizar y regular las condiciones laborales en las nuevas circunstancias productivas (Carrillo et al., 1992).

En este mismo estudio en el que se analizaron 281 plantas maquiladoras, se encontró que el $28 \%$ se podía clasificar como altamente flexible, en función de los métodos y técnicas de organización; sin embargo, no hubo diferencia de estos aspectos entre las plantas con uso de equipo de alta o baja tecnología, lo que evidencia que no hay una asociación obligada entre el desarrollo tecnológico y la flexibilidad laboral (Carrillo et al., 1992).

El hecho de que los cambios organizativos y la flexibilidad laboral ocurran no significa que los trabajadores se encuentren frente a una mejora en sus condiciones al interior del proceso laboral y que los riesgos, así como las exigencias disminuyan. Los cambios se hacen con la finalidad de incrementar la productividad; la flexibilidad añade la polivalencia de las actividades y aumenta, desde la óptica empresarial, la capacidad de adaptación de los trabajadores. Pero ello no necesariamente mejora el contenido del trabajo, ni permite recuperar el saber de los obreros, ni les posibilita el control sobre el proceso laboral. Esta polivalencia, en muchos casos mantiene condiciones de trabajo igual de descalificadas y vacías de contenido que las anteriores. Incluso, la flexibilidad pretende introducir un nuevo desposeimiento en los trabajadores - y esto puede ser considerado una tendencia general del modelo - que con- 
siste en despojarlos de sus condiciones de defensa colectiva afectando, entre otras cosas, las posibilidades de control sobre la nocividad laboral.

Así, las tendencias a limitar o suprimir a los sindicatos, modificar sustancialmente las contrataciones colectivas, individualizar la relación obrero-patronal al interior de cada empresa e, incluso, suprimir la participación del Estado en su papel regulador, son muestra de un mayor resquebrajamiento de esta defensa colectiva.

\section{La mujer y la flexibilización del trabajo}

Una de las principales características de la manufactura para la exportación, en países como México, es su fuerte dependencia del trabajo femenino (Fernández, 1986). Si bien esta situación se ha modificado a lo largo de los años, ya que la participación de los hombres en esta industria ha experimentado un importante aumento en términos absolutos y relativos en los últimos años, la mujer trabajadora sigue participando de manera predominante. Como ya se señaló, dos de cada tres trabajadores de la maquila son mujeres.

La incorporación de la mujer al trabajo de la maquila ha sido ampliamente discutida, reconociéndose diversas razones de naturaleza política y económica. Ha sido frecuente la denuncia de las difíciles condiciones laborales a que se enfrentan y se han cuestionado las políticas del Estado que al impulsar la maquila, han desmantelado las conquistas laborales en casi todos sus aspectos. También se ha planteado que la falta de experiencia organizativa de las trabajadoras ha permitido imponer condiciones laborales por debajo de los límites alcanzados en otros sectores. La participación de la mujer ha llamado la atención no sólo por las condiciones laborales en que se encuentra dentro de la industria, sino también por las condiciones de vida en general y por las responsabilidades que asume en la vida familiar (Garduño, M., et al, 1993; 244). Si bien el análisis de la problemática de la doble jornada en la mujer rebasa los límites de este documento, cabe mencionar que en la IME y en otras industrias en que se ha impulsado la organización flexible del trabajo, las capacidades, aptitudes y habilidades históricamente aprendidas son funcionales al proyecto modernizador. Así, la mujer tiene gran capacidad para convertirse dentro de la fábrica en trabajadora polivalente pues su experiencia familiar de atender múltiples actividades y roles en el hogar le permite integrarse de una manera más inmediata al proceso productivo. De hecho las características anatómicas o psicológicas que esgrimen los gerentes de personal, al referirse a la paciencia y habilidad manual de las trabajadoras, son expresiones de un fenómeno más complejo.

La incorproración de la mujer al trabajo también ha presentado otros puntos de conflicto. El acoso sexual es un hecho común que padecen las trabajadoras, lo cual generalmente no se denuncia por el temor a represalias que pueden llegar al despido (La Botz, D., 1994; 403). Asimismo se ha reconocido la presencia de menores de edad, quiénes para obtener el empleo entregan documentos alterados y por lo mismo temen defender sus derechos laborales. Algunos patrones han reconocido la existencia del trabajo de menores de edad, fenómeno que puede alcanzar hasta el $5 \%$ en la maquila (La Botz, 1994).

\section{El proceso de trabajo en las maquiladoras. Riesgos, exigencias laborales y daños a la salud}

Los estudios que exploran las condiciones de salud de los trabajadores de la maquila han analizado algunos aspectos del proceso de trabajo y los factores de riesgo existentes. Ya se ha mencionado que en la actualidad la IME muestra un panorama heterogéneo de condiciones tecnológicas y formas de organización laboral. Así, se pueden encontrar los procesos típicamente tayloristas con uso intensivo de mano de obra descalificada; otros con tecnología avanzada y aquéllos en los que destaca la organización flexible del proceso laboral. Pero ya se mencionó que también se presenta la conjunción de diversas formas de organización laboral y desarrollo tecnológico.

Si bien no se cuenta con la información suficiente que permita caracterizar con toda precisión los diferentes procesos de trabajo y sus perfiles de riesgos y exigencias, se cuenta con datos que permiten aproximarse a ello. Hacia 1991 , cerca del $20 \%$ de las maquiladoras participaban en el proceso de modernización tecnológico-organizativa. En el sector electrónico, por ejemplo, habían pasado del ensamble manual a la inserción automática. La estructura ocupacional también se había transformado, aumentando la composición de personal calificado con más experiencia laboral y mayor antigüedad (Carrillo et al., 1992).

Sin embargo, una parte significativa - aún con los cambios tecnológicos realizados - con- 
serva elementos importantes de los procesos tradicionales. No es de extrañar que aún existan procesos en los que las tareas son simples y con poca variedad de movimientos ejecutados a altos ritmos de trabajo. En la maquila electrónica dedicada al ensamble de conductores o semiconductores, una operadora coloca 4 terminales en una placa en 4 segundos, es decir, 3,600 componentes en una hora y 28,800 por jornada diaria de trabajo (Márquez et al., 1988). Asimismo en el proceso laboral de la confección de ropa, junto a operaciones que se realizan con maquinaria computarizada, predominan las operaciones con maquinaria tradicional. En este proceso se requieren hasta 50 operaciones para fabricar una prenda y se encuentran ciclos de trabajo de menos de 20 segundos que involucran varias operaciones (Villegas, 1995).

Cabe señalar además, que si bien los procesos de flexibilización del trabajo han cambiado algunos aspectos de la organización laboral al modificar el contenido del trabajo, lo cierto es que estos cambios, supuestamente para enriquecerlo, no han significado necesariamente que la intensidad del mismo disminuya. Lo que ocurre es que la flexibilización del trabajo atiende más al incremento de la productividad y no al mejoramiento de las condiciones laborales. La lectura de los contratos colectivos de trabajo y de los reglamentos internos, como ya se señaló, también lo demuestra.

Así, pese a la diversidad de ramas implicadas en la industria maquiladora, la organización taylorista del trabajo es una de las características centrales de este tipo de procesos. Aún destaca la división extrema del trabajo de tal forma que cada trabajador ejecuta un trabajo manual más o menos simple. Pero en muchas ocasiones este trabajo incluye tareas que requieren de minuciosidad, paciencia y dedicación (Quintero, 1989).

En la industria maquiladora el contenido y la intensidad del trabajo siguen siendo variables problemáticas por las exigencias que implican. En principio la actividad que se realiza no permite ejercitar la capacidad intelectual de los obreros. Por otra parte, la elevada intensidad del trabajo es una característica casi universal de la maquila. Permanentemente se ejerce presión para cumplir con un estándar de producción del cual no hay manera de librarse. El salario y la posibilidad de conservar el empleo dependen de la productividad de los trabajadores. Los supervisores y los jefes de línea, entre otras de sus funciones, vigilan el cumplimiento de ese mínimo de producción establecido.
Estos aspectos han sido detectados en estudios recientes realizados a través de encuestas aplicadas a trabajadores y trabajadoras de la maquila. Llama la atención que, si bien en algunos casos se aprecian cambios en la organización del trabajo, persisten porcentajes importantes de trabajadores que tienen baja escolaridad, no han recibido capacitación para el trabajo, laboran bajo cuotas de producción establecidas y no rotan actividades (Balcazar et al., 1995). Asimismo otro estudio reporta que la existencia de exigencias relacionadas con la organización del trabajo son más críticas para trabajadoras de la maquila que para trabajadoras del sector servicios (Guendelman et al., 1993).

Existen otros ejemplos que ilustran cómo los cambios en la organización del trabajo se han realizado sólo para endosar a los trabajadores la responsabilidad de incrementar la productividad. En estos casos las tradicionales líneas de producción ahora se han convertido en "grupos de trabajo", pero sus miembros siguen realizando tareas parcializadas, monótonas, repetitivas y lo que se ha colectivizado es la responsabilidad de cubrir la cuota de producción, que está fijada para el grupo. De esta manera ahora se busca que los trabajadores se supervisen entre ellos, y lo que resulta aún más contradictorio es que si alguien falta al trabajo, todos son responsables de cubrir la tarea y de alcanzar la cuota de producción del grupo, de lo contrario nadie alcanza sus bonos (Sedepac; $1995 ; 50)$. También se ha reportado que frecuentemente la rotación de puestos en el proceso de trabajo implica realizar tareas más pesadas y repetitivas (Sánchez, 1994).

Frente a los intensos ritmos de trabajo, resulta aún más problemática la existencia de extensas jornadas de trabajo sin que se brinden posibilidades de descanso o recuperación a lo largo de las mismas. En diversos estudios se ha reportado que la duración de la jornada laboral es superior a las 40 horas semanales; algunos reportan promedios de más de 65 horas a la semana, incluyendo alrededor de 15 horas-extra por semana (Denman, 1990; Balcazar et al., 1995); otros reportan alrededor de 48 horas, pero más extensas que las jornadas existentes en el sector servicios (Guendelman et al., 1993); también señalan que hay pausas de 20 minutos al día, en dos momentos de 10 minutos cada uno (Sánchez, 1994).

La información que existe acerca de la morbilidad de los trabajadores de la maquila muestra un panorama contradictorio. Si bien en diversos sentidos los datos muestran una situación crítica, otros estudios encuentran una mejor situación para las trabajadoras de la ma- 
quila al compararlas con otras trabajadoras o amas de casa (Guendelman et al., 1993; Jasis et al., 1993; Melbourne et al., 1988). Sin embargo, algunos autores que reconocen esta situación contradictoria, señalan que al comparar la información de las condiciones de salud entre trabajadores de los países en los que las empresas multinacionales operan, los resultados serían muy diferentes (McGuiness, 1994). De cualquier manera un primer comentario válido en torno a la salud de los trabajadores de la maquila sería para reconocer la insuficiente información al respecto. En las siguientes líneas señalaremos aquellos hallazgos que muestran un panorama crítico y plantean la necesidad de emprender acciones en torno al mejoramiento de las condiciones de trabajo y salud de los trabajadores de la maquila.

Como ya se mencionó, el primer punto que es necesario reconocer es la falta de información que cubra al conjunto de trabajadores y procesos, tanto como riesgos y exigencias laborales existentes. Se han señalado algunas limitaciones en los estudios epidemiológicos realizados, ya que es probable que incluyan un sesgo en los hallazgos obtenidos. Así, el efecto del trabajador sano puede estar influyendo de diversas maneras. Por un lado, la rigurosa selección de personal deja fuera de la maquila a trabajadores que no cumplen los requisitos establecidos por las empresas, por lo que nos encontramos con una población en principio sana, que además es muy joven. Otro punto es la expulsión temprana de la fuerza de trabajo, como ya se dijo, en la IME existe una muy elevada rotación de personal. Es probable que parte de este fenómeno se deba a motivos de salud, lo cual obstaculiza identificar los daños y establecer su asociación con el trabajo, sobre todo cuando los padecimientos tradicionalmente reconocidos por los servicios de salud aparecen después de muchos años de exposición (Melbourne et al., 1988).

En cuanto a los daños a la salud, uno de los aspectos que resulta particularmente importante en la maquila es la problemática de las mujeres en torno a la salud reproductiva. Se han señalado efectos nocivos que incluyen abortos y alteraciones a la salud en los hijos de las trabajadoras (Denman, 1990). Es de notar en primera instancia, que las repercusiones del trabajo no sólo impactan a las obreras sino a sus familias. Ya algunos estudios en otros países refieren tasas elevadas de abortos entre trabajadoras de procesos de la maquila electrónica. Asimismo, el bajo peso al nacer (BPN) es "reflejo de los procesos específicos, particulares y generales en que viven las familias de los recién nacidos" (Denman, 1990). En este problema se han identificado diversos factores de riesgo que atañen a la ocupación de la madre: duración de la jornada, esfuerzo físico y postura, así como exposición a riesgos físicos, químicos y biológicos. Ya en otros estudios en Francia, (refiere Denman) se encontró un índice de prematurez mayor en obreras no clasificadas que en empleadas administrativas o ejecutivas, $8.3 \%$ y $3.8 \%$ respectivamente. Los resultados de la Tabla 2, muestran los hallazgos del estudio realizado en obreras de la maquila de Nogales, en el que se puede observar que el BPN alcanza un índice de $14 \%$, mientras que para las trabajadoras de servicios y comercio es de 5\%; es decir las obreras de la maquila tienen un riesgo de 2.8 veces mayor. Cabe señalar además que en el análsis multivariado que se realizó se encontró que en el caso de las obreras de la maquila, la antigüedad en el trabajo es una variable importante en la explicación del fenómeno, mientras que en el otro grupo ésta no resultó significativa (Denman, 1990).

En otros estudios realizados con información del Instituto Mexicano del Seguro Social, se ha identificado que entre los trabajadores de la maquila no se reportan enfermedades profesionales, pero se encontraron datos interesantes en los expedientes médicos de ese servicio. En primer lugar, llaman la atención las cifras elevadas de demanda de consulta. Así, en el caso de dos empresas estudiadas de la electrónica, se reportaron tasas de 246.3 y 247.4 por cien años-persona (Márquez et al., 1988); para el caso de una procesadora de mariscos, la tasa correspondiente fue de 441 (Carlesso et al., 1985).

Si comparamos estos resultados con reportes de otros estudios basados en la misma fuente, pero realizados en población industrial no perteneciente a la maquiladora (Noriega et al., 1993), donde la tasa de morbilidad general fué de 93.2, podemos observar que la razón de morbilidad es al menos dos, y hasta cuatro veces más alta en los trabajadores de la maquila.

En esos estudios se analizaron también aquellas patologías que pueden asociarse al trabajo. Es interesante mencionar que entre estos padecimientos, los asociados al estrés ocuparon el primer lugar de las causas de consulta. Para las trabajadoras de la maquila de mariscos, los trastornos psíquicos y psicosomáticos representan casi el $50 \%$ de la morbilidad reportada a través de los expedientes de la seguridad social. En la maquila electrónica, predominan los trastornos relacionados con exigencias mentales, tales como la neurosis, cefalea tensional, gastritis, colitis, hipertensión, mialgias y neuralgias. Asimismo, también se 
destacan las enfermedades relacionadas con esfuerzo físico y posiciones incómodas tales como las lumbalgias y fatiga muscular; además son importantes los trastornos oculares y de la piel. Llama la atención que la morbilidad ocular, en procesos con alta demanda visual en el trabajo, disminuye conforme aumenta la antigüedad, lo que permite suponer una expulsión de la fuerza de trabajo deteriorada a partir de los cinco años de estar laborando (Márquez et al., 1988).

Los resultados arrojados por estos estudios, particularmente los relacionados con trastornos psíquicos y psicosomáticos, contrastan notablemente con la información oficial proporcionada por la seguridad social. En esas estadísticas se diagnosticó un promedio de 9 casos de neurosis por año durante el período de 1984 a 1994. Es evidente que estas cifras, que se refieren a la población asegurada de todo el país y que representaron en 1994 el $0.3 \%$ del total de enfermedades profesionales contabilizadas, no reflejan lo que está ocurriendo entre los tabajadores (IMSS, 1995).

El poco reconocimiento a esta problemática ha quedado establecida en el propio Programa de Salud Mental desde hace más de una década “...dentro de los aspectos laborales, una buena parte de las ausencias es resultado de alcoholismo y padecimientos neuróticos que en la mayoría de los casos no son considerados enfermedades incapacitantes que ameritan atención especializada" (Secretaría de Salud, 1985). Sin duda alguna, aún resta mucho por hacer en este terreno, más aún cuando el panorama laboral muestra las tendencias que aquí se han analizado.

Por otra parte, otros autores han mencionado problemas de tipo músculo-esquelético relacionados con las condiciones de trabajo. En ello intervienen problemas de tipo ergonómico como el diseño de los puestos y las herramientas, pero también los elevados ritmos de trabajo y las largas jornadas (La Botz, 1994). Entre otros problemas, el síndrome del túnel carpiano llama la atención porque tampoco aparece reportado en las estadísticas de enfermedades laborales, no obstante que las condiciones existentes en las maquilas indican el elevado riesgo de tal problema. En otros países ha llamado la atención por su alta incidencia y sus consecuencias altamente incapacitantes en procesos taylorizados (Maeno et al., 1995). En encuestas colectivas aplicadas a trabajadoras de la maquila éste es uno de los problemas detectados como de mayor importancia y ha sido reconocido como una causa de abandono de trabajo. Es además, uno de los problemas que

\begin{tabular}{|c|c|c|c|c|}
\hline \multicolumn{5}{|c|}{$\begin{array}{l}\text { Distribución de peso al nacer de hijos de trabajadoras de Nogales, Sonora, } \\
\text { según empleo de la madre. }\end{array}$} \\
\hline \multirow[t]{2}{*}{ peso al nacer (gramos) } & \multicolumn{2}{|c|}{ maquiladora } & \multicolumn{2}{|c|}{ servicios y comercio } \\
\hline & $n$ & $\%$ & $\mathrm{n}$ & $\%$ \\
\hline $\begin{array}{l}\text { bajo peso al nacer } \\
\text { ( } 2500 \text { o menos) }\end{array}$ & 23 & 14 & 6 & 5 \\
\hline $\begin{array}{l}\text { deficiente peso } \\
(2501-2999)\end{array}$ & 23 & 14 & 29 & 22 \\
\hline $\begin{array}{l}\text { peso normal } \\
(3000-3999)\end{array}$ & 114 & 67 & 85 & 65 \\
\hline $\begin{array}{l}\text { alto peso } \\
\text { (4000 o más) }\end{array}$ & 9 & 5 & 11 & 18 \\
\hline total & 169 & 100 & 131 & 100 \\
\hline media & \multicolumn{2}{|c|}{$3193.84 \mathrm{gr}$} & \multicolumn{2}{|c|}{3299.39} \\
\hline D.S. & \multicolumn{2}{|c|}{587.73} & \multicolumn{2}{|c|}{532.00} \\
\hline
\end{tabular}

Fuente: Denman, C. "La salud de las obreras de la maquila. el caso de Sonora". Crisis, conflicto y sobrevivencia. Guadalajara, México. Universidad de Guadalajara, 1990. pp. 229-255

se han discutido en negociaciones colectivas para fijar cuotas y bonos de producción (Sedepac, 1995).

Los riesgos químicos constituyen también un punto de fuerte discusión debido a diversas razones, una de las cuales es el alto número de trabajadoras expuestas. La maquila electrónica, que ocupa un lugar significativo en el empleo, se caracteriza por un alto consumo de sustancias tóxicas que se utilizan en diversas etapas del proceso de limpieza, grabado, afilado, sellado y encapsulado. Los riesgos a la salud se han documentado extensamente en la literatura pero en la IME no se cuenta con un estudio global de exposición ni de daños a la salud (Denman, 1990). Existen denuncias acerca del uso de benceno y el uso indiscriminado, sin los controles necesarios, de otros compuestos químicos entre los que se encuentran otros solventes, metales pesados, etc. (La Botz, 1994).

Otra razón para discutir el impacto de los riesgos químicos trasciende al espacio de las fábricas. La polémica de la contaminación ambiental, el deterioro del medio ambiente y los daños a la salud por ese motivo, es algo que se debate constantemente. Los estudios realizados muestran una situación crítica que ha puesto en riesgo los ecosistemas. Los grupos ecologistas han denunciado numerosas irregularidades que incluyen el contrabando de tóxicos, la eliminación inadecuada de desechos y la existencia de basureros clandestinos. Se reconoce que no existen suficientes plantas de tratamiento y confinamiento adecuados para 
el manejo de residuos tóxicos y peligrosos; incluso existen evidencias de que numerosas empresas eliminan productos químicos a canales, drenajes y depósitos de basura.

En la categoría de generadoras de residuos peligrosos se encuentran 1,035 de las maquilas (Espinosa-Torres et al., 1994). Todos estos puntos llaman la atención acerca de los posibles efectos en la salud de quienes viven en las zonas afectadas, pero también - y eso no ha sido suficientemente valorado como un problema por el conjunto de la sociedad - de quienes trabajan en tales procesos industriales. La doble exposición laboral-ambiental para las trabajadoras de la maquila puede ser un problema importante.

Un aspecto que se debate y sobre el cual aún no existe un dictamen definitivo, es el elevado índice de casos de anencefalia en algunas zonas de la frontera. Entre las causas que podrían estar provocando esta situación se ha mencionado el tráfico de desechos tóxicos a lo largo de la frontera, en el que podrían estar involucradas empresas de la maquila. Lo cierto es que existen numerosas denuncias al respecto e información preocupante, ya que se ha señalado que México tiene las cifras más altas de anencefalia en el mundo al registrar una tasa de 18.4 por cada 10,000 nacimientos, mientras que en Estados Unidos el índice es de 3.0 (El Día, 1993).

Esto llama la atención porque es probable que en un futuro cercano el problema de los tóxicos sea mucho más grave, pues con la firma del TLC algunas disposiciones legales que obligan a las maquiladoras a regresar a los Estados Unidos los residuos tóxicos y peligrosos generados a partir de materia prima estadounidense podrían quedar sin efecto (EspinosaTorres et al., 1994).

Un último aspecto en cuanto a los daños a la salud que queremos señalar es la accidentabilidad en el trabajo. Este fenómeno es el que tradicionalmente llama la atención a los sistemas de seguridad social debido a la compensación de que son objeto. En la actualidad se señalan cifras bajas de accidentes para todo el país, pero de diversas maneras se ha podido apreciar que el problema es mayor de lo que aparece en las estadísticas. En el caso de la maquila las cifras que se han detectado para empresas de la electrónica en Nogales, indican que efectivamente el problema es de gran importancia pues si proyectáramos los datos detectados, a un año de trabajo, la cifra sería de $25,2 \%$ de trabajadores accidentados en ese período, de los cuales más del $40 \%$ produjeron al menos un día de incapacidad. Para el año de estudio, la cifra de los accidentes, "riesgos terminados" que llama el IMSS, fue de $6.4 \%$ (Balcazar et al., 1995). Incluso en otros procesos de la maquila, como el mencionado caso de una empacadora de mariscos, los accidentes fueron la segunda causa de consulta ante el IMSS, con $69 \%$ anual (Carlesso et al., 1985). Sin duda alguna, en este aspecto también distan mucho que desear las condiciones laborales, más aún cuando existe la tendencia al aumento de la intensidad del trabajo.

Otro aspecto que conviene analizar es el que se refiere a las deficientes actividades de salud pública que se realizan en la industria, fenómeno que afecta a los trabajadores de todo el país. En el caso de la IME se destaca que un elevado porcentaje de empresas cuente con servicio médico pero que el personal que lo atiende (médico o enfermera) no ha recibido capacitación en salud laboral (Balcazar et al., 1995). El papel de estos servicios merece una discusión aparte, ya que se ha planteado que intervienen para seleccionar el personal o para evitar que los casos de accidente o enfermedad laboral lleguen a la seguridad social. Así por ejemplo, los criterios para que una trabajadora ingrese a la maquila electrónica están claramente definidos: habilidad manual, buena coordinación óculo-motora y motriz fina, buenos reflejos y agudeza visual, debido a que el trabajo a realizar es minucioso y requiere la manipulación con alto grado de precisión de piezas minúsculas (Márquez et al., 1988). Pero no hay criterios preventivos frente a tal tipo de exigencias: vista fija, alta atención, esfuerzo visual importante. Lo que ocurre cuando aparece un padecimiento, es que el trabajador es expulsado del trabajo. Esto se demuestra cuando se examina el comportamiento epidemiológico de ese padecimiento, ya que la morbilidad disminuye conforme aumenta la antigüedad de las trabajadoras, es decir, la trabajadora que se enferma sale de la fábrica.

\section{Comentario final}

$\mathrm{Al}$ analizar las condiciones laborales y de salud de los trabajadores de la maquila y el efecto que las políticas de modernización han provocado en ellas, se aprecian diversas tendencias que indican cuál es la situación que se espera en los próximos años y se pueden plantear, al menos de manera inicial, algunas alternativas a emprender. Se ha descrito cómo la flexibilización del trabajo con frecuencia da lugar a un abanico más amplio de exigencias para buena parte de los trabajadores, esto como resultado 
del deterioro en la calidad y contenido de trabajo. Los intentos para incrementar la productividad que se han seguido hasta ahora, no han considerado la calidad de las condiciones de trabajo como un elemento fundamental. Es necesario incorporar estos elementos a la discusión, más aún cuando se discutirán nuevas propuestas a la legislación laboral para lo cual los empresarios promoverán el modelo de la IME como prototipo a seguir.

Algunos de los fenómenos que se destacan en los cambios de la organización del trabajo, el aumento de la movilidad laboral y la polivalencia, darán argumentos para desconocer la causalidad de los accidentes y las enfermedades tipificados legalmente como laborales. Pero además, se puede observar que la morbilidad laboral (no sólo la reconocida como tal) se está incrementando. La problemática de los efectos casi inmediatos sobre la salud, debido a la intensidad del trabajo y a los riesgos del proceso, identificados a través del bajo peso al nacer de los hijos de las obreras de la maquila, alerta al respecto. También es claro desde ahora el aumento en las manifestaciones patológicas del estrés y la fatiga.

Bajo ese contexto también parece más crítico el combate frontal a la participación organizada de los trabajadores que se ha apreciado en el medio laboral en general y en la maquila en particular. De no revertirse esta tendencia habrá menos posibilidades de transformar estas condiciones. Cuando analizamos la capacidad de respuesta y de acción en salud de las trabajadoras de la maquila, se hace evidente la necesidad de buscar alternativas para consolidar formas de participación. La vía sindical,

\section{Referencias}

BALCAZAR, H.; DENMAN, C. \& LARA, F., 1995. Factors associated with work-related accidents and sickness among maquiladora workers: the case of Nogales, Sonora, México. International Journal of Health Services, 25:489-502.

CARLESSO, E. \& RODRIGUEZ, J., 1985. Proceso Laboral y el Desgaste Obrero. Caso Maquiladora de Procesamiento de Mariscos en Matamoros. Tesis de Maestría, Xochimilco: Universidad Autónoma Metropolitana.

CARRILLO, J. \& DE LA O, E., 1992. La reestructuración en la industria maquiladora. El cotidiano, 46:5459. parece aún más difícil en este sector de trabajadores que tradicionalmente han enfrentado difíciles condiciones de organización. Sin embargo, la discusión amplia y colectiva, acerca de las condiciones de trabajo y de salud de las maquiladoras parece un punto esencial. Desde luego, una de las tareas para el futuro es involucrar a los diferentes sectores de la sociedad en la discusión y la búsqueda de soluciones. En principio, es necesario difundir la información en torno a los riesgos a la salud que se enfrentan en el trabajo. El debate en torno a la contaminación ambiental y el papel que en ello juegan las maquiladoras, da oportunidad de llamar la atención acerca de los procesos que al interior de las fábricas se generan y los riesgos a que se exponen los trabajadores de esos procesos. Pero la discusión no debe restringirse a los problemas relacionados con los tóxicos, los riesgos físicos y de accidentabilidad, es necesario destacar la organización del proceso laboral y las exigencias que de ella se derivan. Asimismo, los aspectos ergonómicos en este tipo de industrias deben ser discutidos.

Sin duda alguna se debe profundizar en el estudio de la salud laboral en la maquila. La evaluación, incluso de los riesgos "tradicionales" como los contaminantes químicos, aún dista mucho de ser satisfactoria. La elaboración y revisión de normas de exposición a los diferentes riesgos y exigencias de la actividad es un elemento necesario de llevar a cabo, máxime cuando en otras regiones, en el contexto de la globalización económica, sí se están incorporando estos aspectos, definiendo e instrumentando políticas de salud de los trabajadores.

DENMAN, C.,1990. La salud de las obreras de la maquila: el caso de Nogales, Sonora. In: Crisis, Conflicto y Sobrevivencia (G. De la Peña, ed.), pp. 229255. Guadalajara: Universidad de Guadalajara.

EL DIA, 1993. Nota Informativa. 25 de Octubre:18.

ESPINOSA-TORRES, F; HERNANDEZ-AVILA, M. \& LOPEZ-CARRILLO, L., 1994. El TLC: un reto y una oportunidad para la salud ambiental. El caso de las maquiladoras. Salud Pública de México. 36: 597-616.

FERNANDEZ, P., 1986. Asia y Frontera México-Estados Unidos. In: Reestructuración Industrial ( $\mathrm{J}$ Carrillo, org.), pp. 128-143, México: COLEF. 
GARDUÑO, M. A. \& MARQUEZ, M., 1993. Salud Laboral Femenina. Apuntes para su Investigación. In: Para la Investigación sobre la Salud de los Trabajadores, PALTEX, no 3, pp. 235-263. Washington: Oficina Sanitaria Panamericana.

GONZALEZ-ARECHIGA, B. \& J.C. RAMIREZ. 1990. Apertura y Reestructuración en la Maquiladora. México: COLEF.

GUENDELMAN, S. \& JASIS, M. 1993. The health consequences of maquiladora work: women on the US-Mexican border. International Journal of Health Services. 83:37-44.

IMSS (Instituto Mexicano del Seguro Social), 1995. Riesgos de Trabajo e Invalidez. México: Dirección de Prestaciones Médicas, Coordinación de Salud en el Trabajo.

INEGI (Instituto Nacional de Estadística Geografía e Informática), 1994. El ABC de la Estadística de la Industria Maquiladora de Exportación. México: INEGI.

JASIS, M. \& GUENDELMAN, S. 1993. Maquiladoras y mujeres fronterizas: ¿beneficio o daño a la salud obrera?. Salud Pública de México. 35:620-629.

KOPINAK, K. 1994. Maquilización en la Industria Automotriz. Conferencia II sobre Autos y Continentalismo. México.

LA BOTZ, D. 1994. Manufacturing poverty: the maquiladorization of Mexico. International Journal of Health Services. 24:403-408.

MAENO, M.; OLIVEIRA, F.; BONETTI, A.; PEDROLLO, M.; FONSECA, L. \& CAPPUCCI, C., 1995. Reintroduction of Patients Suffering from Overuse Syndrome in The Job Market. Pilot Project Report. São Paulo: Health Secretary of São Paulo.
MARQUEZ, M. \& J. ROMERO. 1988. El desgaste de las obreras de la maquila eléctrico-electrónica. $S a$ lud Problema 14: 9-24.

McGUINNESS, M., 1994. Free trade and occupational health policy: an argument for health and safety across the north american workplace. Salud Pública de México. 36:578-596.

MELBOURNE, F.; SIPAN, C.; HOFSTETTER, R.; DUBOIS, B.; KREFFT, A.; CONWAY, J.; JASIS, M. \& ISAACS, H., 1988. Occupational health risks for mexican women: the case of the maquiladora along the Mexican-United States border. International Journal of Health services, 18:617-627.

NORIEGA, M.; VILLEGAS, J. \& CUELLAR, R. 1993. Las condiciones de salud de los trabajadores industriales de Naucalpan, México. IV Congreso $\mathrm{Na}$ cional de Investigación en Salud Pública. Cuernavaca: IMSS.

QUINTERO, C. 1990. La Sindicalización en las Maquiladoras Tijuanenses. Conaculta: México.

SANCHEZ, S. 1994. El nuevo sindicalismo y la productividad de las maquiladoras de Ciudad Juárez de Chihuahua. El Cotidiano, 64:77-85.

SECRETARIA DE SALUD. 1985. Programa de Salud Mental 1985-1986. México: Subdirección de Normas de Salud Mental.

SEDEPAC. 1995. Memorias del Taller de Salud en el Trabajo en la Maquila. México: Servicio, Desarrollo y Paz, A.C.

VILLEGAS, J., 1995. Job Demands and Musculoskeletal Complaints in Repetitive Work at the Manufacturing Industry. Final report. Xochimilco: Universidad Autónoma Metropolitana. 